\title{
Four periodic solutions for a food-limited two-species Gilpin-Ayala type predator-prey system with harvesting terms on time scales
}

\section{Hui Fang ${ }^{*}$ and Yun Wang}

"Correspondence:

kmustfanghui@hotmail.com Department of Mathematics, Kunming University of Science and Technology, Kunming, Yunnan 650500 , China

\begin{abstract}
By using Mawhin's coincidence degree theory, this paper establishes a new criterion on the existence of four periodic solutions for a food-limited two-species Gilpin-Ayala type predator-prey system with harvesting terms on time scales. An example is given to illustrate the effectiveness of the result.
\end{abstract}

\section{Introduction}

The theory of calculus on time scales was initiated by Hilger [1] in order to unify continuous and discrete analysis, and it has become an effective approach to the study of mathematical models involving the hybrid discrete-continuous processes. Since the population dynamics in the real world usually involves the hybrid discrete-continuous processes, it may be more realistic to consider population models on time scales [2].

In recent years, some researchers studied the existence of periodic solutions for some population models on time scales under the assumption of periodicity of the parameters by using Mawhin's coincidence degree theory (see [3-7]). To our best knowledge, few papers deal with the existence of multiple periodic solutions for population models with harvesting terms on time scales. The main difficulty is that the techniques used in continuous population models with harvesting terms are generally not available to population models with harvesting terms on time scales. Indeed, almost all papers involving continuous population models with harvesting terms used Fermat's theorem on local extrema of differentiable functions in real analysis; for example, see [8-11]. However, Fermat's theorem is not true in time scales calculus.

In this paper, we consider a food-limited two-species Gilpin-Ayala type predator-prey system with harvesting terms on time scales:

$$
\left\{\begin{array}{l}
u_{1}^{\Delta}(t)=\frac{1}{k_{1}(t)+c_{1}(t) e^{u_{1}(t)}}\left[a_{1}(t)-a_{11}(t) e^{\theta_{1} u_{1}(t)}-a_{12}(t) e^{\theta_{2} u_{2}(t)}\right]-\frac{H_{1}(t)}{e^{u_{1}(t)}} \\
u_{2}^{\Delta}(t)=\frac{1}{k_{2}(t)+c_{2}(t) e^{u_{2}(t)}}\left[a_{2}(t)+a_{21}(t) e^{\theta_{1} u_{1}(t)}-a_{22}(t) e^{\theta_{2} u_{2}(t)}\right]-\frac{H_{2}(t)}{e^{u_{2}(t)}} .
\end{array}\right.
$$

In system (1.1), let $x_{i}(t)=\exp \left\{u_{i}(t)\right\}, i=1,2$. If the time scale $\mathbb{T}=\mathbb{R}$ (the set of all real numbers), then system (1.1) reduces to

$$
\left\{\begin{array}{l}
x_{1}^{\prime}(t)=\frac{x_{1}(t)}{k_{1}(t)+c_{1}(t) x_{1}(t)}\left[a_{1}(t)-a_{11}(t) x_{1}^{\theta_{1}}(t)-a_{12}(t) x_{2}^{\theta_{2}}(t)\right]-H_{1}(t), \\
x_{2}^{\prime}(t)=\frac{x_{2}(t)}{k_{2}(t)+c_{2}(t) x_{2}(t)}\left[a_{2}(t)+a_{21}(t) x_{1}^{\theta_{1}}(t)-a_{22}(t) x_{2}^{\theta_{2}}(t)\right]-H_{2}(t),
\end{array}\right.
$$

๑) 2013 Fang and Wang; licensee Springer. This is an Open Access article distributed under the terms of the Creative Commons Attribution License (http://creativecommons.org/licenses/by/2.0), which permits unrestricted use, distribution, and reproduction in any medium, provided the original work is properly cited. 
where $x_{1}$ and $x_{2}$ denote the prey and the predator, respectively; $a_{i}(t)(i=1,2), a_{i i}(t)(i=1,2)$, $H_{i}(t)(i=1,2)$ are all positive continuous functions denoting the intrinsic growth rate, the intra-specific competition rates and the harvesting rates, respectively; $a_{12}(t)$ is the predation rate of the predator and $a_{21}(t)$ represents the conversion rate; $k_{i}(t)(i=1,2)$ are the population numbers of two species at saturation, respectively. $\theta_{i}(i=1,2)$ represent a nonlinear measure of interspecific interference. When $c_{i}(t) \neq 0(i=1,2), \frac{a_{i}(t)}{k_{i}(t) c_{i}(t)}(i=1,2)$ are the rate of replacement of mass in the population at saturation (including the replacement of metabolic loss and of dead organisms). In this case, system (1.2) is a food-limited population model. For other food-limited population models, we refer to [12-17] and the references cited therein. When $\theta_{i} \neq 1(i=1,2)$, system (1.2) is a Gilpin-Ayala type population model. Gilpin-Ayala type population models were firstly proposed by Gilpin and Ayala in [18]. For some recent work, we refer to [17, 19-22]. When $c_{i}(t) \equiv 0, \theta_{i}=1(i=1,2)$, system (1.2) was consider by Zhao and Ye [9].

Motivated by the work of Bohner et al. [3] and Chen [8], we study the existence of multiple periodic solutions of (1.1) by using Mawhin's coincidence degree.

\section{Preliminaries from calculus on time scales}

In this section, we briefly present some foundational definitions and results from the calculus on time scales so that the paper is self-contained. For more details, one can see $[1,23,24]$.

Definition 2.1 [23] A time scale $\mathbb{T}$ is an arbitrary nonempty closed subset of the real numbers $\mathbb{R}$.

Let $\omega>0$. Throughout this paper, the time scale $\mathbb{T}$ is assumed to be $\omega$-periodic, i.e., $t \in \mathbb{T}$ implies $t+\omega \in \mathbb{T}$. In particular, the time scale $\mathbb{T}$ under consideration is unbounded above and below.

Definition 2.2 [23] We define the forward jump operator $\sigma: \mathbb{T} \rightarrow \mathbb{T}$, the backward jump operator $\rho: \mathbb{T} \rightarrow \mathbb{T}$, and the graininess $\mu: \mathbb{T} \rightarrow \mathbb{R}^{+}=[0,+\infty)$ by

$$
\sigma(t):=\inf \{s \in \mathbb{T}: s>t\}, \quad \rho(t):=\sup \{s \in \mathbb{T}: s<t\}, \quad \mu(t)=\sigma(t)-t \quad \text { for } t \in \mathbb{T},
$$

respectively. If $\sigma(t)=t$, then $t$ is called right-dense (otherwise, right-scattered), and if $\rho(t)=t$, then $t$ is called left-dense (otherwise, left-scattered).

Definition 2.3 [23] Assume that $f: \mathbb{T} \rightarrow \mathbb{R}$ is a function, and let $t \in \mathbb{T}$. Then we define $f^{\Delta}(t)$ to be the number (provided it exists) with the property that given any $\varepsilon>0$, there is a neighborhood $U$ of $t$ (i.e., $U=(t-\delta, t+\delta) \cap \mathbb{T}$ for some $\delta>0$ ) such that

$$
\left|[f(\sigma(t))-f(s)]-f^{\Delta}(t)[\sigma(t)-s]\right| \leq \varepsilon|\sigma(t)-s| \quad \text { for all } s \in U .
$$

In this case, $f^{\Delta}(t)$ is called the delta (or Hilger) derivative of $f$ at $t$. Moreover, $f$ is said to be delta or Hilger differentiable on $\mathbb{T}$ if $f^{\Delta}(t)$ exists for all $t \in \mathbb{T}$.

Definition 2.4 [23] A function $F: \mathbb{T} \rightarrow \mathbb{R}$ is called an antiderivative of $f: \mathbb{T} \rightarrow \mathbb{R}$ provided $F^{\Delta}(t)=f(t)$ for all $t \in \mathbb{T}$. Then we define

$$
\int_{r}^{s} f(t) \Delta t=F(s)-f(r) \quad \text { for } r, s \in \mathbb{T} .
$$


Definition 2.5 [23] A function $f: \mathbb{T} \rightarrow \mathbb{R}$ is said to be rd-continuous if it is continuous at right-dense points in $\mathbb{T}$ and its left-sided limits exist (finite) at left-dense points in $\mathbb{T}$. The set of rd-continuous functions $f: \mathbb{T} \rightarrow \mathbb{R}$ will be denoted by $C_{\text {rd }}(\mathbb{T})$.

The following notation will be used throughout this paper.

Let

$$
\begin{aligned}
& \mathbb{T}^{\kappa}= \begin{cases}\mathbb{T} \backslash(\rho(\sup \mathbb{T}), \sup \mathbb{T}], & \text { if } \sup \mathbb{T}<+\infty, \\
\mathbb{T}, & \text { if } \sup \mathbb{T}=+\infty,\end{cases} \\
& \kappa=\min \{[0,+\infty) \cap \mathbb{T}\}, \quad I_{\omega}=[\kappa, \kappa+\omega] \cap \mathbb{T}, \quad g^{u}=\sup _{t \in I_{\omega}} g(t), \quad g^{l}=\inf _{t \in I_{\omega}} g(t), \\
& \bar{g}=\frac{1}{\omega} \int_{I_{\omega}} g(s) \Delta s=\frac{1}{\omega} \int_{\kappa}^{\kappa+\omega} g(s) \Delta s,
\end{aligned}
$$

where $g \in C_{\mathrm{rd}}(\mathbb{T})$ is a nonnegative $\omega$-periodic real function, i.e., $g(t+\omega)=g(t)$ for all $t \in \mathbb{T}$.

Lemma 2.1 [23] Every rd-continuous function has an antiderivative.

Lemma 2.2 [23] Assume that $f: \mathbb{T} \rightarrow \mathbb{R}$ is a function, and let $t \in \mathbb{T}^{\kappa}$. Then we have:

(i) Iff is continuous at $t$ and $t$ is right-scattered, then $f$ is differential at $t$ with

$$
f^{\Delta}(t)=\frac{f(\sigma(t))-f(t)}{\mu(t)}
$$

(ii) Iff is right-dense, then $f$ is differential at $t$ iff the limit

$$
\lim _{s \rightarrow t} \frac{f(t)-f(s)}{t-s}
$$

exists as a finite number. In this case,

$$
f^{\Delta}(t)=\lim _{s \rightarrow t} \frac{f(t)-f(s)}{t-s}
$$

Lemma 2.3 [3] Let $t_{1}, t_{2} \in I_{\omega}$ and $t \in \mathbb{T}$. If $g: \mathbb{T} \rightarrow \mathbb{R}$ is $\omega$-periodic, then

$$
g(t) \leq g\left(t_{1}\right)+\int_{\kappa}^{\kappa+\omega}|g(s)| \Delta s \quad \text { and } \quad g(t) \geq g\left(t_{2}\right)-\int_{\kappa}^{\kappa+\omega}|g(s)| \Delta s .
$$

Lemma 2.4 [6] Assume that $\left\{f_{n}\right\}_{n \in N}$ is a function sequence on $I_{\omega}$ such that

(i) $\left\{f_{n}\right\}_{n \in N}$ is uniformly bounded on $I_{\omega}$;

(ii) $\left\{f_{n}^{\Delta}\right\}_{n \in N}$ is uniformly bounded on $I_{\omega}$.

Then there is a subsequence of $\left\{f_{n}\right\}_{n \in N}$ converging uniformly on $I_{\omega}$.

\section{Existence of multiple periodic solutions}

We first briefly state Mawhin's coincidence degree theory (see [25]).

Let $X, Z$ be normed vector spaces, $L: \operatorname{dom} L \subset X \rightarrow Z$ be a linear mapping, $N: X \rightarrow Z$ be a continuous mapping. The mapping $L$ will be called a Fredholm mapping of index zero if $\operatorname{dim} \operatorname{Ker} L=\operatorname{codim} \operatorname{Im} L<+\infty$ and $\operatorname{Im} L$ is closed in $Z$. If $L$ is a Fredholm mapping of index zero, then there exist continuous projectors (i.e., linear and idempotent linear operators) 
$P: X \rightarrow X$ and $Q: Z \rightarrow Z$ such that $\operatorname{Im} P=\operatorname{Ker} L, \operatorname{Im} L=\operatorname{Ker} Q=\operatorname{Im}(I-Q)$. If we define $L_{P}: \operatorname{dom} L \cap \operatorname{Ker} P \rightarrow \operatorname{Im} L$ as the restriction $\left.L\right|_{\operatorname{dom} L \cap \operatorname{Ker} P}$ of $L$ to $\operatorname{dom} L \cap \operatorname{Ker} P$, then $L_{P}$ is invertible. We denote the inverse of that map by $K_{P}$. If $\Omega$ is an open bounded subset of $X$, the mapping $N$ will be called $L$-compact on $\bar{\Omega}$ if $Q N(\bar{\Omega})$ is bounded and $K_{P}(I-Q) N: \bar{\Omega} \rightarrow$ $X$ is compact, i.e., continuous and such that $K_{P}(I-Q) N(\bar{\Omega})$ is relatively compact. Since $\operatorname{Im} Q$ is isomorphic to $\operatorname{Ker} L$, there exists an isomorphism $J: \operatorname{Im} Q \rightarrow \operatorname{Ker} L$.

For convenience, we introduce Mawhin's continuation theorem [25] as follows.

Lemma 3.1 Let $L$ be a Fredholm mapping of index zero, and let $N: \bar{\Omega} \times[0,1] \rightarrow Z$ be L-compact on $\bar{\Omega} \times[0,1]$. Suppose

(a) $L u \neq \lambda N(u, \lambda)$ for every $u \in \operatorname{dom} L \cap \partial \Omega$ and every $\lambda \in(0,1)$;

(b) $Q N(u, 0) \neq 0$ for every $u \in \partial \Omega \cap \operatorname{Ker} L$;

(c) Brouwer degree $\operatorname{deg}_{B}\left(\left.\operatorname{JQN}(\cdot, 0)\right|_{\operatorname{Ker} L}, \Omega \cap \operatorname{Ker} L, 0\right) \neq 0$.

Then $L u=N(u, 1)$ has at least one solution in $\operatorname{dom} L \cap \bar{\Omega}$.

Set

$$
h(x)=b-a x^{\alpha}-\frac{c}{x}, \quad x \in(0,+\infty) .
$$

Lemma 3.2 [17] Assume that $a, b, c, \alpha$ are positive constants and

$$
b>(1+\alpha) a^{\frac{1}{1+\alpha}}\left(\frac{c}{\alpha}\right)^{\frac{\alpha}{1+\alpha}} .
$$

Then there exist $0<x^{-}<x^{+}$such that

$$
\begin{aligned}
& h\left(x^{-}\right)=h\left(x^{+}\right)=0, \quad h(x)>0 \quad \text { for } x \in\left(x^{-}, x^{+}\right), \\
& h(x)<0 \quad \text { for } x \in\left(0, x^{-}\right) \cup\left(x^{+},+\infty\right)
\end{aligned}
$$

and

$$
h^{\prime}\left(x^{-}\right)>0, \quad h^{\prime}\left(x^{+}\right)<0 .
$$

Set

$$
\begin{aligned}
& N_{1}=\frac{\ln \left(\frac{a_{1}}{a_{11}}\right)^{u}}{\theta_{1}}+2 \omega\left(\frac{a_{1}}{k_{1}}\right)^{u}, \\
& N_{2}=\frac{\ln \left[\left(\frac{a_{2}}{a_{22}}\right)^{u}+\left(\frac{a_{21}}{a_{22}}\right)^{u} e^{\theta_{1} N_{1}}\right]}{\theta_{2}}+2 \omega\left(\frac{a_{2}}{k_{2}}\right)^{u}+2 \omega\left(\frac{a_{21}}{k_{2}}\right)^{u} e^{\theta_{1} N_{1}} .
\end{aligned}
$$

From now on, we always assume that:

$\left(\mathrm{H}_{1}\right) c_{i}(t), k_{i}(t), a_{i}(t), H_{i}(t)(i=1,2), a_{i j}(t)(i, j=1,2)$ are positive continuous $\omega$-periodic functions, $\theta_{i}(i=1,2)$ are positive constants.

$\left(\mathrm{H}_{2}\right) \frac{1}{1+\left(\frac{c_{1}}{k_{1}}\right)^{u} e^{N_{1}}}\left[\left(\frac{a_{1}}{k_{1}}\right)^{l}-\left(\frac{a_{12}}{k_{1}}\right)^{u} e^{\theta_{2} N_{2}}\right]>\left(1+\theta_{1}\right)\left[\left(\frac{a_{11}}{k_{1}}\right)^{u}\right]^{\frac{1}{1+\theta_{1}}}\left[\frac{H_{1}^{u}}{\theta_{1}}\right]^{\frac{\theta_{1}}{1+\theta_{1}}}$.

$\left(\mathrm{H}_{3}\right) \frac{\left(\frac{a_{2}}{k_{2}}\right)^{l}}{1+\left(\frac{c_{2}}{k_{2}}\right)^{u} e^{N_{2}}}>\left(1+\theta_{2}\right)\left[\left(\frac{a_{22}}{k_{2}}\right)^{u}\right]^{\frac{1}{1+\theta_{2}}}\left[\frac{H_{2}^{u}}{\theta_{2}}\right]^{\frac{\theta_{2}}{1+\theta_{2}}}$. 
Set

$$
\begin{aligned}
& M_{1}(x)=\frac{1}{1+\left(\frac{c_{1}}{k_{1}}\right)^{u} e^{N_{1}}}\left[\left(\frac{a_{1}}{k_{1}}\right)^{l}-\left(\frac{a_{12}}{k_{1}}\right)^{u} e^{\theta_{2} N_{2}}\right]-\left(\frac{a_{11}}{k_{1}}\right)^{u} x^{\theta_{1}}-\frac{H_{1}^{u}}{x}, \\
& M_{2}(x)=\frac{\left(\frac{a_{2}}{k_{2}}\right)^{l}}{1+\left(\frac{c_{2}}{k_{2}}\right)^{u} e^{N_{2}}}-\left(\frac{a_{22}}{k_{2}}\right)^{u} x^{\theta_{2}}-\frac{H_{2}^{u}}{x}, \\
& p_{i}(x)=\overline{\left(\frac{a_{i}}{k_{i}}\right)}-\overline{\left(\frac{a_{i i}}{k_{i}}\right)} x^{\theta_{i}}-\frac{\bar{H}_{i}}{x} \quad(i=1,2), \\
& m_{1}(x)=\left(\frac{a_{1}}{k_{1}}\right)^{u}-\frac{\left(\frac{a_{11}}{k_{1}}\right)^{l}}{1+\left(\frac{c_{1}}{k_{1}}\right)^{u}\left[\left(\frac{a_{1}}{a_{11}}\right)^{u}\right]^{\frac{1}{\theta_{1}}}} x^{\theta_{1}}-\frac{H_{1}^{l}}{x}, \\
& m_{2}(x)=\left(\frac{a_{2}}{k_{2}}\right)^{u}+\left(\frac{a_{21}}{k_{2}}\right)^{u} e^{\theta_{1} N_{1}}-\frac{\left(\frac{a_{22}}{k_{2}}\right)^{l}}{1+\left(\frac{c_{2}}{k_{2}}\right)^{u}\left[\left(\frac{a_{2}}{a_{22}}\right)^{u}+\left(\frac{a_{21}}{a_{22}}\right)^{u} e^{\left.\theta_{1} N_{1}\right]^{\frac{1}{\theta_{2}}}}\right.} x^{\theta_{2}}-\frac{H_{2}^{l}}{x} .
\end{aligned}
$$

Lemma 3.3 Assume that $\left(\mathrm{H}_{1}\right)-\left(\mathrm{H}_{3}\right)$ hold. Then the following assertions hold:

(1) There exist $0<u_{i}^{-}<u_{i}^{+}$such that

$$
M_{i}\left(u_{i}^{-}\right)=M_{i}\left(u_{i}^{+}\right)=0, \quad M_{i}(x)>0 \quad \text { for } x \in\left(u_{i}^{-}, u_{i}^{+}\right)
$$

and

$$
M_{i}(x)<0 \quad \text { for } x \in\left(0, u_{i}^{-}\right) \cup\left(u_{i}^{+},+\infty\right), i=1,2 \text {. }
$$

(2) There exist $0<x_{i}^{-}<x_{i}^{+}$such that

$$
\begin{aligned}
& p_{i}\left(x_{i}^{-}\right)=p_{i}\left(x_{i}^{+}\right)=0, \quad p_{i}(x)>0 \quad \text { for } x \in\left(x_{i}^{-}, x_{i}^{+}\right), \\
& p_{i}(x)<0 \quad \text { for } x \in\left(0, x_{i}^{-}\right) \cup\left(x_{i}^{+},+\infty\right)
\end{aligned}
$$

and

$$
p_{i}^{\prime}\left(x_{i}^{-}\right)>0, \quad p_{i}^{\prime}\left(x_{i}^{+}\right)<0, \quad i=1,2 .
$$

(3) There exist $0<l_{i}^{-}<l_{i}^{+}$such that

$$
m_{i}\left(l_{i}^{-}\right)=m_{i}\left(l_{i}^{+}\right)=0, \quad m_{i}(x)>0 \quad \text { for } x \in\left(l_{i}^{-}, l_{i}^{+}\right)
$$

and

$$
m_{i}(x)<0 \quad \text { for } x \in\left(0, l_{i}^{-}\right) \cup\left(l_{i}^{+},+\infty\right), i=1,2 \text {. }
$$

(4)

$$
l_{i}^{-}<x_{i}^{-}<u_{i}^{-}<u_{i}^{+}<x_{i}^{+}<l_{i}^{+}, \quad i=1,2 .
$$


(5)

$$
\ln u_{i}^{+}<N_{i}, \quad i=1,2 \text {. }
$$

Proof It follows from $\left(\mathrm{H}_{1}\right)-\left(\mathrm{H}_{3}\right)$ and Lemma 3.2 that assertions (1)-(3) hold. Noticing that

$$
\begin{aligned}
& \frac{1}{1+\left(\frac{c_{1}}{k_{1}}\right)^{u} e^{N_{1}}}\left[\left(\frac{a_{1}}{k_{1}}\right)^{l}-\left(\frac{a_{12}}{k_{1}}\right)^{u} e^{\theta_{2} N_{2}}\right] \overline{\left(\frac{a_{1}}{k_{1}}\right)} \leq\left(\frac{a_{1}}{k_{1}}\right)^{u}, \\
& \frac{\left(\frac{a_{11}}{k_{1}}\right)^{l}}{1+\left(\frac{c_{1}}{k_{1}}\right)^{u}\left[\left(\frac{a_{1}}{a_{11}}\right)^{u}\right]^{\frac{1}{\theta_{1}}}}<\overline{\left(\frac{a_{11}}{k_{1}}\right)} \leq\left(\frac{a_{11}}{k_{1}}\right)^{u}, \\
& \frac{\left(\frac{a_{2}}{k_{2}}\right)^{l}}{1+\left(\frac{c_{2}}{k_{2}}\right)^{u} e^{N_{2}}}<\overline{\left(\frac{a_{2}}{k_{2}}\right)}<\left(\frac{a_{2}}{k_{2}}\right)^{u}+\left(\frac{a_{21}}{k_{2}}\right)^{u} e^{\theta_{1} N_{1}}, \\
& \frac{\left(\frac{a_{22}}{k_{2}}\right)^{l}}{1+\left(\frac{c_{2}}{k_{2}}\right)^{u}\left[\left(\frac{a_{2}}{a_{22}}\right)^{u}+\left(\frac{a_{21}}{a_{22}}\right)^{u} e^{\left.\theta_{1} N_{1}\right]^{\frac{1}{\theta_{2}}}}<\left(\frac{a_{22}}{k_{2}}\right)\right.} \leq\left(\frac{a_{22}}{k_{2}}\right)^{u}, \\
& H_{i}^{l} \leq \bar{H}_{i} \leq H_{i}^{u} \quad(i=1,2),
\end{aligned}
$$

we have

$$
M_{i}(x)<p_{i}(x)<m_{i}(x), \quad i=1,2 .
$$

By assertions (1)-(3), assertion (4) holds.

It follows from $M_{i}\left(u_{i}^{+}\right)=0(i=1,2)$ that

$$
\begin{aligned}
& \left(\frac{a_{11}}{k_{1}}\right)^{u}\left[u_{1}^{+}\right]^{\theta_{1}}<\frac{1}{1+\left(\frac{c_{1}}{k_{1}}\right)^{u} e^{N_{1}}}\left[\left(\frac{a_{1}}{k_{1}}\right)^{l}-\left(\frac{a_{12}}{k_{1}}\right)^{u} e^{\theta_{2} N_{2}}\right]<\left(\frac{a_{1}}{k_{1}}\right)^{l}, \\
& \left(\frac{a_{22}}{k_{2}}\right)^{u}\left[u_{2}^{+}\right]^{\theta_{2}}<\frac{\left(\frac{a_{2}}{k_{2}}\right)^{l}}{1+\left(\frac{c_{2}}{k_{2}}\right)^{u} e^{N_{2}}}<\left(\frac{a_{2}}{k_{2}}\right)^{l} .
\end{aligned}
$$

Therefore, we have

$$
\begin{aligned}
& u_{1}^{+}<\left[\frac{\left(\frac{a_{1}}{k_{1}}\right)^{l}}{\left(\frac{a_{11}}{k_{1}}\right)^{u}}\right]^{\frac{1}{\theta_{1}}} \leq\left[\left(\frac{a_{1}}{a_{11}}\right)^{u}\right]^{\frac{1}{\theta_{1}}}<e^{N_{1}}, \\
& u_{2}^{+}<\left[\frac{\left(\frac{a_{2}}{k_{2}}\right)^{l}}{\left(\frac{a_{22}}{k_{2}}\right)^{u}}\right]^{\frac{1}{\theta_{2}}} \leq\left[\left(\frac{a_{2}}{a_{22}}\right)^{u}\right]^{\frac{1}{\theta_{2}}}<e^{N_{2}},
\end{aligned}
$$

which implies that assertion (5) also holds.

Now, we are ready to state the main result of this paper.

Theorem 3.1 Assume that $\left(\mathrm{H}_{1}\right)-\left(\mathrm{H}_{3}\right)$ hold. Then system (1.1) has at least four $\omega$-periodic solutions.

Proof Take

$$
X=Z=\left\{u=\left(u_{1}, u_{2}\right)^{T}: u_{i} \in C_{\mathrm{rd}}\left(\mathbb{T}, \mathbb{R}^{2}\right) \text { and } u_{i}(t+\omega)=u_{i}(t) \text { for all } t \in \mathbb{T}, i=1,2\right\}
$$


and define

$$
\|u\|=\left[\sum_{i=1}^{2}\left(\max _{t \in I_{\omega}}\left|u_{i}(t)\right|\right)^{2}\right]^{\frac{1}{2}}, \quad u \in X(\text { or } Z) .
$$

Equipped with the above norm $\|\cdot\|$, it is easy to verify that $X$ and $Z$ are both Banach spaces.

Set

$$
\begin{aligned}
\Delta_{1}(u, t, \lambda)= & {\left[\frac{k_{1}(t)+(1-\lambda) c_{1}(t) e^{u_{1}(t)}}{k_{1}(t)+c_{1}(t) e^{u_{1}(t)}}\right] } \\
& \times\left[\frac{a_{1}(t)}{k_{1}(t)}-\frac{a_{11}(t) e^{\theta_{1} u_{1}(t)}}{k_{1}(t)}-\frac{\lambda a_{12}(t) e^{\theta_{2} u_{2}(t)}}{k_{1}(t)}\right]-\frac{H_{1}(t)}{e^{u_{1}(t)}}, \\
\Delta_{2}(u, t, \lambda)= & {\left[\frac{k_{2}(t)+(1-\lambda) c_{2}(t) e^{u_{2}(t)}}{k_{2}(t)+c_{2}(t) e^{u_{2}(t)}}\right] } \\
& \times\left[\frac{a_{2}(t)}{k_{2}(t)}+\frac{\lambda a_{21}(t) e^{\theta_{1} u_{1}(t)}}{k_{2}(t)}-\frac{a_{22}(t) e^{\theta_{2} u_{2}(t)}}{k_{2}(t)}\right]-\frac{H_{2}(t)}{e^{u_{2}(t)}} .
\end{aligned}
$$

Define the mappings $L: X \rightarrow Z, N: X \times[0,1] \rightarrow Z, P: X \rightarrow X$ and $Q: Z \rightarrow Z$ as follows:

$$
\begin{aligned}
& N(u, \lambda)=\left(\begin{array}{l}
\Delta_{1}(u, t, \lambda) \\
\Delta_{2}(u, t, \lambda)
\end{array}\right), \\
& L u=\left(\begin{array}{l}
u_{1}^{\Delta} \\
u_{2}^{\Delta}
\end{array}\right), \quad P u=\frac{1}{\omega} \int_{\kappa}^{\kappa+\omega} u(t) \Delta t=Q u, \quad u \in X(\text { or } Z) .
\end{aligned}
$$

We first show that $L$ is a Fredholm mapping of index zero and $N$ is $L$-compact on $\bar{\Omega} \times[0,1]$ for any open bounded set $\Omega \subset X$. The argument is standard, one can see [3-5]. But for the sake of completeness, we give the details here.

It is easy to see that $\operatorname{Ker} L=\left\{u \in X:\left(u_{1}(t), u_{2}(t)\right)^{T}=\left(h_{1}, h_{2}\right)^{T} \in \mathbb{R}^{2}\right.$ for $\left.t \in \mathbb{T}\right\}, \operatorname{Im} L=$ $\left\{u \in X: \int_{\kappa}^{\kappa+\omega} u(t) \Delta t=0\right\}$ is closed in $Z$, and $\operatorname{dim} \operatorname{Ker} L=\operatorname{codim} \operatorname{Im} L=2$. Therefore, $L$ is a Fredholm mapping of index zero. Clearly, $P$ and $Q$ are continuous projectors such that

$$
\operatorname{Im} P=\operatorname{Ker} L, \quad \operatorname{Ker} Q=\operatorname{Im} L=\operatorname{Im}(I-Q) .
$$

On the other hand, $K_{p}: \operatorname{Im} L \rightarrow \operatorname{dom} L \cap \operatorname{Ker} P$, the inverse to $L$, exists and is given by

$$
K_{p}(u)=\int_{\kappa}^{t} u(s) \Delta s-\frac{1}{\omega} \int_{\kappa}^{\kappa+\omega} \int_{\kappa}^{\eta} u(s) \Delta s \Delta \eta .
$$

Obviously, $Q N$ and $K_{p}(I-Q) N$ are continuous. By Lemma 2.4, it is not difficult to show that $\overline{K_{p}(I-Q) N(\bar{\Omega} \times[0,1])}$ is compact for any open bounded set $\Omega \subset X$. Moreover, $Q N(\bar{\Omega} \times[0,1])$ is bounded. Hence, $N$ is $L$-compact on $\bar{\Omega} \times[0,1]$ for any open bounded set $\Omega \subset X$.

In order to apply Lemma 3.1, we need to find at least four appropriate open, bounded subsets $\Omega_{1}, \Omega_{2}, \Omega_{3}, \Omega_{4}$ in $X$. 
Corresponding to the operator equation $L u=\lambda N(u, \lambda), \lambda \in(0,1)$, we have

$$
\begin{aligned}
u_{1}^{\Delta}(t)= & \lambda\left[\frac{k_{1}(t)+(1-\lambda) c_{1}(t) e^{u_{1}(t)}}{k_{1}(t)+c_{1}(t) e^{u_{1}(t)}}\right] \\
& \times\left[\frac{a_{1}(t)}{k_{1}(t)}-\frac{a_{11}(t) e^{\theta_{1} u_{1}(t)}}{k_{1}(t)}-\frac{\lambda a_{12}(t) e^{\theta_{2} u_{2}(t)}}{k_{1}(t)}\right]-\frac{\lambda H_{1}(t)}{e^{u_{1}(t)}}, \\
u_{2}^{\Delta}(t)= & \lambda\left[\frac{k_{2}(t)+(1-\lambda) c_{2}(t) e^{u_{2}(t)}}{k_{2}(t)+c_{2}(t) e^{u_{2}(t)}}\right] \\
& \times\left[\frac{a_{2}(t)}{k_{2}(t)}+\frac{\lambda a_{21}(t) e^{\theta_{1} u_{1}(t)}}{k_{2}(t)}-\frac{a_{22}(t) e^{\theta_{2} u_{2}(t)}}{k_{2}(t)}\right]-\frac{\lambda H_{2}(t)}{e^{u_{2}(t)}} .
\end{aligned}
$$

Suppose that $\left(u_{1}(t), u_{2}(t)\right)^{T}$ is an $\omega$-periodic solution of $(3.1),(3.2)$ for some $\lambda \in(0,1)$. Since $u(t)=\left(u_{1}(t), u_{2}(t)\right) \in X$, there exist $t_{i}^{M}, t_{i}^{m} \in[\kappa, \kappa+\omega] \cap \mathbb{T}, i=1,2$, such that

$$
\begin{aligned}
& u_{i}\left(t_{i}^{M}\right)=\max _{t \in[\kappa, \kappa+\omega] \cap \mathbb{T}} u_{i}(t), \\
& u_{i}\left(t_{i}^{m}\right)=\min _{t \in[\kappa, \kappa+\omega] \cap \mathbb{T}} u_{i}(t), \quad i=1,2 .
\end{aligned}
$$

By Lemma 2.2, it is easy to see that

$$
u_{i}^{\Delta}\left(t_{i}^{M}\right) \leq 0, \quad u_{i}^{\Delta}\left(t_{i}^{m}\right) \geq 0, \quad i=1,2 .
$$

From this and (3.1), (3.2), we obtain that

$$
\begin{aligned}
0 \geq & {\left[\frac{k_{1}\left(t_{1}^{M}\right)+(1-\lambda) c_{1}\left(t_{1}^{M}\right) e^{u_{1}\left(t_{1}^{M}\right)}}{\left.k_{1}\left(t_{1}^{M}\right)+c_{1}\left(t_{1}^{M}\right) e^{u_{1}\left(t_{1}^{M}\right)}\right]}\right.} \\
& \times\left[\frac{a_{1}\left(t_{1}^{M}\right)}{k_{1}\left(t_{1}^{M}\right)}-\frac{a_{11}\left(t_{1}^{M}\right) e^{\theta_{1} u_{1}\left(t_{1}^{M}\right)}}{k_{1}\left(t_{1}^{M}\right)}-\frac{\lambda a_{12}\left(t_{1}^{M}\right) e^{\theta_{2} u_{2}\left(t_{1}^{M}\right)}}{k_{1}\left(t_{1}^{M}\right)}\right]-\frac{H_{1}\left(t_{1}^{M}\right)}{e^{u_{1}\left(t_{1}^{M}\right)}}, \\
0 \geq & {\left[\frac{k_{2}\left(t_{2}^{M}\right)+(1-\lambda) c_{2}\left(t_{2}^{M}\right) e^{u_{2}\left(t_{2}^{M}\right)}}{\left.k_{2}\left(t_{2}^{M}\right)+c_{2}\left(t_{2}^{M}\right) e^{u_{2}\left(t_{2}^{M}\right)}\right]}\right.} \\
& \times\left[\frac{a_{2}\left(t_{2}^{M}\right)}{k_{2}\left(t_{2}^{M}\right)}+\frac{\lambda a_{21}\left(t_{2}^{M}\right) e^{\theta_{1} u_{1}\left(t_{2}^{M}\right)}}{k_{2}\left(t_{2}^{M}\right)}-\frac{a_{22}\left(t_{2}^{M}\right) e^{\theta_{2} u_{2}\left(t_{2}^{M}\right)}}{k_{2}\left(t_{2}^{M}\right)}\right]-\frac{H_{2}\left(t_{2}^{M}\right)}{e^{u_{2}\left(t_{2}^{M}\right)}}
\end{aligned}
$$

and

$$
\begin{aligned}
& 0 \leq\left[\frac{k_{1}\left(t_{1}^{m}\right)+(1-\lambda) c_{1}\left(t_{1}^{m}\right) e^{u_{1}\left(t_{1}^{m}\right)}}{k_{1}\left(t_{1}^{m}\right)+c_{1}\left(t_{1}^{m}\right) e^{u_{1}\left(t_{1}^{m}\right)}}\right] \\
& \times\left[\frac{a_{1}\left(t_{1}^{m}\right)}{k_{1}\left(t_{1}^{m}\right)}-\frac{a_{11}\left(t_{1}^{m}\right) e^{\theta_{1} u_{1}\left(t_{1}^{m}\right)}}{k_{1}\left(t_{1}^{m}\right)}-\frac{\lambda a_{12}\left(t_{1}^{m}\right) e^{\theta_{2} u_{2}\left(t_{1}^{m}\right)}}{k\left(t_{1}^{m}\right)}\right]-\frac{H_{1}\left(t_{1}^{m}\right)}{e^{u_{1}\left(t_{1}^{m}\right)}}, \\
& 0 \leq\left[\frac{k_{2}\left(t_{2}^{m}\right)+(1-\lambda) c_{2}\left(t_{2}^{m}\right) e^{u_{2}\left(t_{2}^{m}\right)}}{k_{2}\left(t_{2}^{m}\right)+c_{2}\left(t_{2}^{m}\right) e^{u_{2}\left(t_{2}^{m}\right)}}\right] \\
& \times\left[\frac{a_{2}\left(t_{2}^{m}\right)}{k_{2}\left(t_{2}^{m}\right)}+\frac{\lambda a_{21}\left(t_{2}^{m}\right) e^{\theta_{1} u_{1}\left(t_{2}^{m}\right)}}{k_{2}\left(t_{2}^{m}\right)}-\frac{a_{22}\left(t_{2}^{m}\right) e^{\theta_{2} u_{2}\left(t_{2}^{m}\right)}}{k_{2}\left(t_{2}^{m}\right)}\right]-\frac{H_{2}\left(t_{2}^{m}\right)}{e^{u_{2}\left(t_{2}^{m}\right)}} .
\end{aligned}
$$




\section{Claim A}

$$
\begin{aligned}
& u_{1}\left(t_{1}^{M}\right)<N_{1}:=\frac{\ln \left(\frac{a_{1}}{a_{11}}\right)^{u}}{\theta_{1}}+2 \omega\left(\frac{a_{1}}{k_{1}}\right)^{u}, \\
& u_{2}\left(t_{2}^{M}\right)<N_{2}:=\frac{\ln \left[\left(\frac{a_{2}}{a_{22}}\right)^{u}+\left(\frac{a_{21}}{a_{22}}\right)^{u} e^{\theta_{1} N_{1}}\right]}{\theta_{2}}+2 \omega\left(\frac{a_{2}}{k_{2}}\right)^{u}+2 \omega\left(\frac{a_{21}}{k_{2}}\right)^{u} e^{\theta_{1} N_{1}} .
\end{aligned}
$$

From (3.1), we obtain that

$$
\begin{aligned}
\int_{\kappa}^{\kappa+\omega} & {\left[\frac{k_{1}(t)+(1-\lambda) c_{1}(t) e^{u_{1}(t)}}{k_{1}(t)+c_{1}(t) e^{u_{1}(t)}}\right] \frac{a_{1}(t)}{k_{1}(t)} \Delta t } \\
= & \int_{\kappa}^{\kappa+\omega}\left[\frac{k_{1}(t)+(1-\lambda) c_{1}(t) e^{u_{1}(t)}}{k_{1}(t)+c_{1}(t) e^{u_{1}(t)}}\right]\left[\frac{a_{11}(t) e^{\theta_{1} u_{1}(t)}}{k_{1}(t)}+\frac{\lambda a_{12}(t) e^{\theta_{2} u_{2}(t)}}{k_{1}(t)}\right] \Delta t \\
& +\int_{\kappa}^{\kappa+\omega} \frac{H_{1}(t)}{e^{u_{1}(t)}} \Delta t .
\end{aligned}
$$

Therefore, we have

$$
\begin{aligned}
& \int_{\kappa}^{\kappa+\omega}\left|u_{1}^{\Delta}(t)\right| \Delta t \\
& \quad<\int_{\kappa}^{\kappa+\omega}\left[\frac{k_{1}(t)+(1-\lambda) c_{1}(t) e^{u_{1}(t)}}{k_{1}(t)+c_{1}(t) e^{u_{1}(t)}}\right]\left[\frac{a_{1}(t)}{k_{1}(t)}+\frac{a_{11}(t) e^{\theta_{1} u_{1}(t)}}{k_{1}(t)}+\frac{\lambda a_{12}(t) e^{\theta_{2} u_{2}(t)}}{k_{1}(t)}\right] \Delta t \\
& \quad+\int_{\kappa}^{\kappa+\omega} \frac{H_{1}(t)}{e^{u_{1}(t)}} \Delta t \\
& =2 \int_{\kappa}^{\kappa+\omega}\left[\frac{k_{1}(t)+(1-\lambda) c_{1}(t) e^{u_{1}(t)}}{k_{1}(t)+c_{1}(t) e^{u_{1}(t)}}\right] \frac{a_{1}(t)}{k_{1}(t)} \Delta t \\
& <2 \int_{\kappa}^{\kappa+\omega} \frac{a_{1}(t)}{k_{1}(t)} \Delta t \leq 2 \omega\left(\frac{a_{1}}{k_{1}}\right)^{u} .
\end{aligned}
$$

By (3.5), we have

$$
\frac{a_{11}\left(t_{1}^{m}\right) e^{\theta_{1} u_{1}\left(t_{1}^{m}\right)}}{k_{1}\left(t_{1}^{m}\right)}<\frac{a_{1}\left(t_{1}^{m}\right)}{k_{1}\left(t_{1}^{m}\right)}
$$

which implies

$$
e^{\theta_{1} u_{1}\left(t_{1}^{m}\right)}<\frac{a_{1}\left(t_{1}^{m}\right)}{a_{11}\left(t_{1}^{m}\right)} \leq\left(\frac{a_{1}}{a_{11}}\right)^{u} .
$$

Therefore, we have

$$
u_{1}\left(t_{1}^{m}\right)<\frac{\ln \left(\frac{a_{1}}{a_{11}}\right)^{u}}{\theta_{1}}
$$

From (3.7), (3.8) and Lemma 2.3, we have

$$
u_{1}\left(t_{1}^{M}\right) \leq u_{1}\left(t_{1}^{m}\right)+\int_{\kappa}^{\kappa+\omega}\left|u_{1}^{\Delta}(t)\right| \Delta t<\frac{\ln \left(\frac{a_{1}}{a_{11}}\right)^{u}}{\theta_{1}}+2 \omega\left(\frac{a_{1}}{k_{1}}\right)^{u}:=N_{1} .
$$


From (3.2), we obtain that

$$
\begin{aligned}
& \int_{\kappa}^{\kappa+\omega}\left[\frac{k_{2}(t)+(1-\lambda) c_{2}(t) e^{u_{2}(t)}}{k_{2}(t)+c_{2}(t) e^{u_{2}(t)}}\right]\left[\frac{a_{2}(t)}{k_{2}(t)}+\frac{\lambda a_{21}(t) e^{\theta_{1} u_{1}(t)}}{k_{2}(t)}\right] \Delta t \\
& =\int_{\kappa}^{\kappa+\omega}\left[\frac{k_{2}(t)+(1-\lambda) c_{2}(t) e^{u_{2}(t)}}{k_{2}(t)+c_{2}(t) e^{u_{2}(t)}}\right] \frac{a_{22}(t) e^{\theta_{2} u_{2}(t)}}{k_{2}(t)} \Delta t+\int_{\kappa}^{\kappa+\omega} \frac{H_{2}(t)}{e^{u_{2}(t)}} \Delta t .
\end{aligned}
$$

Therefore, we have

$$
\begin{aligned}
\int_{\kappa}^{\kappa+\omega} & \left|u_{2}^{\Delta}(t)\right| \Delta t \\
< & \int_{\kappa}^{\kappa+\omega}\left[\frac{k_{2}(t)+(1-\lambda) c_{2}(t) e^{u_{2}(t)}}{k_{2}(t)+c_{2}(t) e^{u_{2}(t)}}\right]\left[\frac{a_{2}(t)}{k_{2}(t)}+\frac{\lambda a_{21}(t) e^{\theta_{1} u_{1}(t)}}{k_{2}(t)}+\frac{a_{22}(t) e^{\theta_{2} u_{2}(t)}}{k_{2}(t)}\right] \Delta t \\
& +\int_{\kappa}^{\kappa+\omega} \frac{H_{2}(t)}{e^{u_{2}(t)}} \Delta t \\
= & 2 \int_{\kappa}^{\kappa+\omega}\left[\frac{k_{2}(t)+(1-\lambda) c_{2}(t) e^{u_{2}(t)}}{k_{2}(t)+c_{2}(t) e^{u_{2}(t)}}\right]\left[\frac{a_{2}(t)}{k_{2}(t)}+\frac{\lambda a_{21}(t) e^{\theta_{1} u_{1}(t)}}{k_{2}(t)}\right] \Delta t \\
< & 2 \int_{\kappa}^{\kappa+\omega}\left[\frac{a_{2}(t)}{k_{2}(t)}+\frac{\lambda a_{21}(t) e^{\theta_{1} u_{1}(t)}}{k_{2}(t)}\right] \Delta t .
\end{aligned}
$$

From this and (3.9), we have

$$
\int_{\kappa}^{\kappa+\omega}\left|u_{2}^{\Delta}(t)\right| \Delta t<2 \omega\left(\frac{a_{2}}{k_{2}}\right)^{u}+2 \omega\left(\frac{a_{21}}{k_{2}}\right)^{u} e^{\theta_{1} N_{1}} .
$$

By (3.6), we have

$$
\frac{a_{22}\left(t_{2}^{m}\right) e^{\theta_{2} u_{2}\left(t_{2}^{m}\right)}}{k_{2}\left(t_{2}^{m}\right)}<\frac{a_{2}\left(t_{2}^{m}\right)}{k_{2}\left(t_{2}^{m}\right)}+\frac{\lambda a_{21}\left(t_{2}^{m}\right) e^{\theta_{1} u_{1}\left(t_{2}^{m}\right)}}{k_{2}\left(t_{2}^{m}\right)},
$$

which implies

$$
e^{\theta_{2} u_{2}\left(t_{2}^{m}\right)}<\frac{a_{2}\left(t_{2}^{m}\right)}{a_{22}\left(t_{2}^{m}\right)}+\frac{a_{21}\left(t_{2}^{m}\right)}{a_{22}\left(t_{2}^{m}\right)} e^{\theta_{1} N_{1}} \leq\left(\frac{a_{2}}{a_{22}}\right)^{u}+\left(\frac{a_{21}}{a_{22}}\right)^{u} e^{\theta_{1} N_{1}} .
$$

Therefore, we have

$$
u_{2}\left(t_{2}^{m}\right)<\frac{\ln \left[\left(\frac{a_{2}}{a_{22}}\right)^{u}+\left(\frac{a_{21}}{a_{22}}\right)^{u} e^{\theta_{1} N_{1}}\right]}{\theta_{2}} .
$$

From (3.10), (3.11) and Lemma 2.3, we have

$$
\begin{aligned}
u_{2}\left(t_{2}^{M}\right) & \leq u_{2}\left(t_{2}^{m}\right)+\int_{\kappa}^{\kappa+\omega}\left|u_{2}^{\Delta}(t)\right| \Delta t \\
& <\frac{\ln \left[\left(\frac{a_{2}}{a_{22}}\right)^{u}+\left(\frac{a_{21}}{a_{22}}\right)^{u} e^{\theta_{1} N_{1}}\right]}{\theta_{2}}+2 \omega\left(\frac{a_{2}}{k_{2}}\right)^{u}+2 \omega\left(\frac{a_{21}}{k_{2}}\right)^{u} e^{\theta_{1} N_{1}}:=N_{2} .
\end{aligned}
$$

\section{Claim B}

$$
\ln l_{i}^{-}<u_{i}\left(t_{i}^{m}\right)<\ln l_{i}^{+}, \quad i=1,2 .
$$


From (3.5) and noticing that

$$
\frac{k_{1}\left(t_{1}^{m}\right)}{k_{1}\left(t_{1}^{m}\right)+c_{1}\left(t_{1}^{m}\right) e^{u_{1}\left(t_{1}^{m}\right)}}<\frac{k_{1}\left(t_{1}^{m}\right)+(1-\lambda) c_{1}\left(t_{1}^{m}\right) e^{u_{1}\left(t_{1}^{m}\right)}}{k_{1}\left(t_{1}^{m}\right)+c_{1}\left(t_{1}^{m}\right) e^{u_{1}\left(t_{1}^{m}\right)}}<1,
$$

we have

$$
0<\left(\frac{a_{1}}{k_{1}}\right)^{u}-\frac{a_{11}\left(t_{1}^{m}\right)}{k_{1}\left(t_{1}^{m}\right)+c_{1}\left(t_{1}^{m}\right) e^{u_{1}\left(t_{1}^{m}\right)}} e^{\theta_{1} u_{1}\left(t_{1}^{m}\right)}-\frac{H_{1}^{l}}{e^{u_{1}\left(t_{1}^{m}\right)}} .
$$

Therefore, by (3.8), we have

$$
0<\left(\frac{a_{1}}{k_{1}}\right)^{u}-\frac{\left(\frac{a_{11}}{k_{1}}\right)^{l}}{1+\left(\frac{c_{1}}{k_{1}}\right)^{u}\left[\left(\frac{a_{1}}{a_{11}}\right)^{u}\right]^{\frac{1}{\theta_{1}}}} e^{\theta_{1} u_{1}\left(t_{1}^{m}\right)}-\frac{H_{1}^{l}}{e^{u_{1}\left(t_{1}^{m}\right)}} .
$$

From assertion (3) of Lemma 3.3 and the above inequality, we have

$$
\ln l_{1}^{-}<u_{1}\left(t_{1}^{m}\right)<\ln l_{1}^{+} .
$$

Similarly, from (3.6) and noticing that

$$
\frac{k_{2}\left(t_{2}^{m}\right)}{k_{2}\left(t_{2}^{m}\right)+c_{2}\left(t_{2}^{m}\right) e^{u_{2}\left(t_{2}^{m}\right)}}<\frac{k_{2}\left(t_{2}^{m}\right)+(1-\lambda) c_{2}\left(t_{2}^{m}\right) e^{u_{2}\left(t_{2}^{m}\right)}}{k_{2}\left(t_{2}^{m}\right)+c_{2}\left(t_{2}^{m}\right) e^{u_{2}\left(t_{2}^{m}\right)}}<1,
$$

we have

$$
0<\left(\frac{a_{2}}{k_{2}}\right)^{u}+\left(\frac{a_{21}}{k_{2}}\right)^{u} e^{\theta_{1} N_{1}}-\frac{a_{22}\left(t_{2}^{m}\right)}{k_{2}\left(t_{2}^{m}\right)+c_{2}\left(t_{2}^{m}\right) e^{u_{2}\left(t_{2}^{m}\right)}} e^{\theta_{2} u_{2}\left(t_{2}^{m}\right)}-\frac{H_{2}^{l}}{e^{u_{2}\left(t_{2}^{m}\right)}} .
$$

Therefore, by (3.11), we have

$$
0<\left(\frac{a_{2}}{k_{2}}\right)^{u}+\left(\frac{a_{21}}{k_{2}}\right)^{u} e^{\theta_{1} N_{1}}-\frac{\left(\frac{a_{22}}{k_{2}}\right)^{l}}{1+\left(\frac{c_{2}}{k_{2}}\right)^{u}\left[\left(\frac{a_{2}}{a_{22}}\right)^{u}+\left(\frac{a_{21}}{a_{22}}\right)^{u} e^{\theta_{1} N_{1}}\right]^{\frac{1}{\theta_{2}}}} e^{\theta_{2} u_{2}\left(t_{2}^{m}\right)}-\frac{H_{2}^{l}}{e^{u_{2}\left(t_{2}^{m}\right)}} .
$$

From assertion (3) of Lemma 3.3 and the above inequality, we have

$$
\ln l_{2}^{-}<u_{2}\left(t_{2}^{m}\right)<\ln l_{2}^{+} .
$$

\section{Claim C}

$$
u_{i}\left(t_{i}^{M}\right)>\ln u_{i}^{+} \quad \text { or } \quad u_{i}\left(t_{i}^{M}\right)<\ln u_{i}^{-}, \quad i=1,2 .
$$

From (3.3), $\left(\mathrm{H}_{2}\right)$ and noticing that

$$
\frac{k_{1}\left(t_{1}^{M}\right)}{k_{1}\left(t_{1}^{M}\right)+c_{1}\left(t_{1}^{M}\right) e^{u_{1}\left(t_{1}^{M}\right)}}<\frac{k_{1}\left(t_{1}^{M}\right)+(1-\lambda) c_{1}\left(t_{1}^{M}\right) e^{u_{1}\left(t_{1}^{M}\right)}}{k_{1}\left(t_{1}^{M}\right)+c_{1}\left(t_{1}^{M}\right) e^{u_{1}\left(t_{1}^{M}\right)}}<1,
$$


we have

$$
0>\frac{k_{1}\left(t_{1}^{M}\right)}{k_{1}\left(t_{1}^{M}\right)+c_{1}\left(t_{1}^{M}\right) e^{u_{1}\left(t_{1}^{M}\right)}}\left[\left(\frac{a_{1}}{k_{1}}\right)^{l}-\left(\frac{a_{12}}{k_{1}}\right)^{u} e^{\theta_{2} N_{2}}\right]-\left(\frac{a_{11}}{k_{1}}\right)^{u} e^{\theta_{1} u_{1}\left(t_{1}^{M}\right)}-\frac{H_{1}^{u}}{e^{u_{1}\left(t_{1}^{M}\right)}} .
$$

Therefore, we have

$$
0>\frac{1}{1+\left(\frac{c_{1}}{k_{1}}\right)^{u} e^{N_{1}}}\left[\left(\frac{a_{1}}{k_{1}}\right)^{l}-\left(\frac{a_{12}}{k_{1}}\right)^{u} e^{\theta_{2} N_{2}}\right]-\left(\frac{a_{11}}{k_{1}}\right)^{u} e^{\theta_{1} u_{1}\left(t_{1}^{M}\right)}-\frac{H_{1}^{u}}{e^{u_{1}\left(t_{1}^{M}\right)}} .
$$

From assertion (1) of Lemma 3.3 and (3.15), we have

$$
u_{1}\left(t_{1}^{M}\right)>\ln u_{1}^{+} \quad \text { or } \quad u_{1}\left(t_{1}^{M}\right)<\ln u_{1}^{-} .
$$

By a similar argument, it follows from (3.4) that

$$
0>\frac{\left(\frac{a_{2}}{k_{2}}\right)^{l}}{1+\left(\frac{c_{2}}{k_{2}}\right)^{u} e^{N_{2}}}-\left(\frac{a_{22}}{k_{2}}\right)^{u} e^{\theta_{2} u_{2}\left(t_{2}^{M}\right)}-\frac{H_{2}^{u}}{e^{u_{2}\left(t_{2}^{M}\right)}} .
$$

From assertion (1) of Lemma 3.3 and (3.17), we have

$$
u_{2}\left(t_{2}^{M}\right)>\ln u_{2}^{+} \quad \text { or } \quad u_{2}\left(t_{2}^{M}\right)<\ln u_{2}^{-} .
$$

It follows from (3.9), (3.13), (3.16) and assertions (4)-(5) of Lemma 3.3 that

$$
\begin{aligned}
& u_{1}\left(t_{1}^{M}\right) \in\left(\ln l_{1}^{-}, \ln u_{1}^{-}\right) \cup\left(\ln u_{1}^{+}, N_{1}\right), \\
& u_{1}\left(t_{1}^{m}\right) \in\left(\ln l_{1}^{-}, \ln l_{1}^{+}\right) .
\end{aligned}
$$

It follows from (3.12), (3.14), (3.18) and assertions (4)-(5) of Lemma 3.3 that

$$
\begin{aligned}
& u_{2}\left(t_{2}^{M}\right) \in\left(\ln l_{2}^{-}, \ln u_{2}^{-}\right) \cup\left(\ln u_{2}^{+}, N_{2}\right), \\
& u_{2}\left(t_{2}^{m}\right) \in\left(\ln l_{2}^{-}, \ln l_{2}^{+}\right) .
\end{aligned}
$$

Clearly, $l_{i}^{ \pm}, u_{i}^{ \pm}(i=1,2)$ are independent of $\lambda$. Now, let us consider $Q N(u, 0)$ with $u=$ $\left(u_{1}, u_{2}\right)^{T} \in \mathbb{R}^{2}$. Note that

$$
Q N(u, 0)=\left(\frac{\overline{\left(\frac{a_{1}}{k_{1}}\right)}-\overline{\left(\frac{a_{11}}{k_{1}}\right)} e^{\theta_{1} u_{1}}-\frac{\bar{H}_{1}}{e^{u_{1}}}}{\left(\frac{a_{2}}{k_{2}}\right)}-\overline{\left(\frac{a_{22}}{k_{2}}\right)} e^{\theta_{2} u_{2}}-\frac{\bar{H}_{2}}{e^{u_{2}}}\right) .
$$

Therefore, it follows from assertion (2) of Lemma 3.3 that $Q N(u, 0)=0$ has four distinct solutions:

$$
\begin{array}{ll}
\tilde{u}_{1}=\left(\ln x_{1}^{+}, \ln x_{2}^{+}\right)^{T}, & \tilde{u}_{2}=\left(\ln x_{1}^{+}, \ln x_{2}^{-}\right)^{T}, \\
\tilde{u}_{3}=\left(\ln x_{1}^{-}, \ln x_{2}^{+}\right)^{T}, & \tilde{u}_{4}=\left(\ln x_{1}^{-}, \ln x_{2}^{-}\right)^{T} .
\end{array}
$$


Let

$$
\begin{aligned}
& \Omega_{1}=\left\{\begin{array}{l|l}
u=\left(u_{1}, u_{2}\right)^{T} \in X & \begin{array}{l}
\max _{t \in I_{\omega}} u_{1}(t) \in\left(\ln u_{1}^{+}, \max \left\{\ln l_{1}^{+}, N_{1}\right\}\right), \\
\min _{t \in I_{\omega}} u_{1}(t) \in\left(\ln l_{1}^{-}, \ln l_{1}^{+}\right), \\
\max _{t \in I_{\omega}} u_{2}(t) \in\left(\ln u_{2}^{+}, \max \left\{\ln l_{2}^{+}, N_{2}\right\}\right), \\
\min _{t \in I_{\omega}} u_{2}(t) \in\left(\ln l_{2}^{-}, \ln l_{2}^{+}\right)
\end{array}
\end{array}\right\}, \\
& \Omega_{2}=\left\{\begin{array}{l|l}
u=\left(u_{1}, u_{2}\right)^{T} \in X & \begin{array}{l}
\max _{t \in I_{\omega}} u_{1}(t) \in\left(\ln u_{1}^{+}, \max \left\{\ln l_{1}^{+}, N_{1}\right\}\right), \\
\min _{t \in I_{\omega}} u_{1}(t) \in\left(\ln l_{1}^{-}, \ln l_{1}^{+}\right), \\
\max _{t \in I_{\omega}} u_{2}(t) \in\left(\ln l_{2}^{-}, \ln u_{2}^{-}\right), \\
\min _{t \in I_{\omega}} u_{2}(t) \in\left(\ln l_{2}^{-}, \ln l_{2}^{+}\right)
\end{array}
\end{array}\right\}, \\
& \Omega_{3}=\left\{\begin{array}{l|l}
u=\left(u_{1}, u_{2}\right)^{T} \in X & \begin{array}{l}
\max _{t \in I_{\omega}} u_{1}(t) \in\left(\ln l_{1}^{-}, \ln u_{1}^{-}\right), \\
\min _{t \in I_{\omega}} u_{1}(t) \in\left(\ln l_{1}^{-}, \ln l_{1}^{+}\right), \\
\max _{t \in I_{\omega}} u_{2}(t) \in\left(\ln u_{2}^{+}, \max \left\{\ln l_{2}^{+}, N_{2}\right\}\right), \\
\min _{t \in I_{\omega}} u_{2}(t) \in\left(\ln l_{2}^{-}, \ln l_{2}^{+}\right)
\end{array}
\end{array}\right\},
\end{aligned}
$$

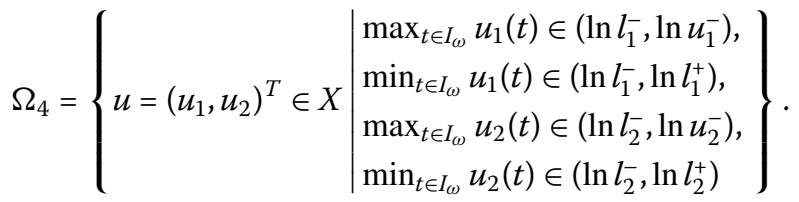

Then $\Omega_{1}, \Omega_{2}, \Omega_{3}, \Omega_{4}$ are bounded open subsets of $X$. It follows from assertion (4) of Lemma 3.3, (3.23) and (3.24) that $\tilde{u}_{i} \in \Omega_{i}(i=1,2,3,4)$. From assertion (4) of Lemma 3.3, (3.19)-(3.22), it is easy to see that $\bar{\Omega}_{i} \cap \bar{\Omega}_{j}=\emptyset(i, j=1,2,3,4, i \neq j)$ and $\Omega_{i}$ satisfies (a) in Lemma 3.1 for $i=1,2,3,4$. Moreover, $Q N(u, 0) \neq 0$ for $u \in \partial \Omega_{i} \cap \operatorname{Ker} L$. By assertion (2) of Lemma 3.3, a direct computation gives

$$
\begin{aligned}
& \operatorname{deg}\left\{\operatorname{JQN}(\cdot, 0), \Omega_{1} \cap \operatorname{Ker} L, 0\right\}=1, \\
& \operatorname{deg}\left\{J Q N(\cdot, 0), \Omega_{2} \cap \operatorname{Ker} L, 0\right\}=-1, \\
& \operatorname{deg}\left\{J Q N(\cdot, 0), \Omega_{3} \cap \operatorname{Ker} L, 0\right\}=-1, \\
& \operatorname{deg}\left\{J Q N(\cdot, 0), \Omega_{4} \cap \operatorname{Ker} L, 0\right\}=1 .
\end{aligned}
$$

Here, $J$ is taken as the identity mapping since $\operatorname{Im} Q=\operatorname{Ker} L$. So far we have proved that $\Omega_{i}$ satisfies all the assumptions in Lemma 3.1. Hence, (1.1) has at least four $\omega$-periodic solutions $\left(u_{1}^{i}(t), u_{2}^{i}(t)\right)^{T}(i=1,2,3,4)$ with $\left(u_{1}^{i}, u_{2}^{i}\right)^{T} \in \operatorname{dom} L \cap \bar{\Omega}_{i}$. Obviously, $\left(u_{1}^{i}(t), u_{2}^{i}(t)\right)^{T}$ $(i=1,2,3,4)$ are different.

Example 3.1 In system (1.1), take

$$
\omega=2, \quad \mathbb{T}_{0}=\{0,0.5\} \cup[1,2], \quad \mathbb{T}=\left\{t_{0}+2 k \mid t_{0} \in \mathbb{T}_{0}, k \in \mathbb{Z}\right\},
$$

where $\mathbb{Z}$ is the integer set. Clearly, the time scale $\mathbb{T}$ is $\omega$-periodic, i.e., $t \in \mathbb{T}$ implies $t+\omega \in \mathbb{T}$. In this case, we have

$$
\kappa=\min \{[0,+\infty) \cap \mathbb{T}\}=0, \quad I_{\omega}=[\kappa, \kappa+\omega] \cap \mathbb{T}=\mathbb{T}_{0}=\{0,0.5\} \cup[1,2] .
$$


Take

$$
\begin{aligned}
& \theta_{1}=\theta_{2}=0.5, \quad H_{1}(t)=\frac{2+\sin (\pi t)}{54,000}, \quad H_{2}(t)=\frac{2+\sin (\pi t)}{3,000}, \\
& k_{1}(t)=100(2+\sin (\pi t)), \quad k_{2}(t)=100(3+\sin (\pi t)), \\
& a_{1}(t)=[2+\sin (\pi t)]^{2}, \quad a_{11}(t)=\frac{[2+\sin (\pi t)]^{2}}{10}, \\
& a_{12}(t)=\frac{[2+\sin (\pi t)]^{2}}{9\left(20+0.5 e^{0.06}\right) e^{\left(0.08+0.002 e^{0.06}\right)},} \\
& a_{2}(t)=[3+\sin (\pi t)]^{2}, \quad a_{22}(t)=\frac{[3+\sin (\pi t)]^{2}}{20}, \quad a_{21}(t)=\frac{[3+\sin (\pi t)]^{2}}{400}, \\
& c_{1}(t)=\frac{2+\sin (\pi t)}{e^{0.12}}, \quad c_{2}(t)=\frac{3+\sin (\pi t)}{\left[2+0.05 e^{0.06}\right]^{2} e^{\left(0.16+0.004 e^{0.06}\right)}} .
\end{aligned}
$$

Then

$$
\begin{aligned}
& H_{1}^{u}=\frac{1}{18,000}, \quad H_{2}^{u}=\frac{1}{1,000}, \\
& \left(\frac{a_{1}}{k_{1}}\right)^{l}=\frac{1}{100}, \quad\left(\frac{a_{1}}{k_{1}}\right)^{u}=\frac{3}{100}, \quad\left(\frac{a_{11}}{k_{1}}\right)^{l}=\frac{1}{1,000}, \quad\left(\frac{a_{11}}{k_{1}}\right)^{u}=\frac{3}{1,000}, \\
& \left(\frac{a_{12}}{k_{1}}\right)^{u}=\frac{1}{300\left(20+0.5 e^{0.06}\right) e^{\left(0.08+0.002 e^{0.06}\right)}}, \\
& \left(\frac{a_{2}}{k_{2}}\right)^{l}=\frac{1}{50}, \quad\left(\frac{a_{2}}{k_{2}}\right)^{u}=\frac{1}{25}, \quad\left(\frac{a_{22}}{k_{2}}\right)^{l}=\frac{1}{1,000}, \\
& \left(\frac{a_{22}}{k_{2}}\right)^{u}=\frac{1}{500}, \quad\left(\frac{a_{21}}{k_{2}}\right)^{u}=\frac{1}{10,000}, \\
& \left(\frac{a_{1}}{a_{11}}\right)^{u}=10, \quad\left(\frac{a_{2}}{a_{22}}\right)^{u}=20, \quad\left(\frac{a_{21}}{a_{22}}\right)^{u}=\frac{1}{20}, \\
& \left(\frac{c_{1}}{k_{1}}\right)^{u}=\frac{1}{100 e^{0.12}}, \quad\left(\frac{c_{2}}{k_{2}}\right)^{u}=\frac{1}{\left[20+0.5 e^{0.06}\right]^{2} e^{\left(0.16+0.004 e^{0.06}\right)}}, \\
& N_{1}=2 \ln 10+0.12, \quad N_{2}=2 \ln \left[20+0.5 e^{0.06}\right]+0.16+0.004 e^{0.06} .
\end{aligned}
$$

Therefore, we have

$$
\begin{aligned}
& \frac{1}{1+\left(\frac{c_{1}}{k_{1}}\right)^{u} e^{N_{1}}}\left[\left(\frac{a_{1}}{k_{1}}\right)^{l}-\left(\frac{a_{12}}{k_{1}}\right)^{u} e^{\theta_{2} N_{2}}\right]=\frac{1}{300}, \quad \frac{\left(\frac{a_{2}}{k_{2}}\right)^{l}}{1+\left(\frac{c_{2}}{k_{2}}\right)^{u} e^{N_{2}}}=\frac{1}{100}, \\
& \left(1+\theta_{1}\right)\left[\left(\frac{a_{11}}{k_{1}}\right)^{u}\right]^{\frac{1}{1+\theta_{1}}}\left[\frac{H_{1}^{u}}{\theta_{1}}\right]^{\frac{\theta_{1}}{1+\theta_{1}}}=1.5 \times\left(\frac{3}{1,000}\right)^{\frac{2}{3}} \times\left(\frac{1}{9,000}\right)^{\frac{1}{3}}=\frac{1.5}{1,000}, \\
& \left(1+\theta_{2}\right)\left[\left(\frac{a_{22}}{k_{2}}\right)^{u}\right]^{\frac{1}{1+\theta_{2}}}\left[\frac{H_{2}^{u}}{\theta_{2}}\right]^{\frac{\theta_{2}}{1+\theta_{2}}}=1.5 \times\left(\frac{1}{500}\right)^{\frac{2}{3}} \times\left(\frac{1}{500}\right)^{\frac{1}{3}}=\frac{3}{1,000} .
\end{aligned}
$$

Hence, all the conditions in Theorem 3.1 are satisfied. By Theorem 3.1, system (1.1) has at least four 2-periodic solutions. 


\section{Competing interests}

The authors declare that they have no competing interests.

\section{Authors' contributions}

All authors contributed equally to the manuscript and read and approved the final manuscript.

\section{Acknowledgements}

This research is supported by the National Natural Science Foundation of China (Grant No. 10971085).

Received: 25 January 2013 Accepted: 23 August 2013 Published: 23 September 2013

\section{References}

1. Hilger, S: Analysis on measure chains - a unified approach to continuous and discrete calculus. Results Math. 18, 8-56 (1990)

2. Gamarra, JGP, Solé, RV: Complex discrete dynamics from simple continuous population models. Bull. Math. Biol. 64, 611-620 (2002)

3. Bohner, M, Fan, M, Zhang, J: Existence of periodic solutions in predator-prey and competition dynamic systems. Nonlinear Anal., Real World Appl. 7, 1193-1204 (2006)

4. Fazly, M, Hesaaraki, M: Periodic solutions for predator-prey systems with Beddington-DeAngelis functional response on time scales. Nonlinear Anal., Real World Appl. 9, 1224-1235 (2008)

5. Zhang, WP, Bi, P, Zhu, DM: Periodicity in a ratio-dependent predator-prey system with stage-structured predator on time scales. Nonlinear Anal., Real World Appl. 9, 344-353 (2008)

6. Xing, Y, Han, M, Zheng, G: Initial value problem for first-order integro-differential equation of Volterra type on time scale. Nonlinear Anal. 60, 429-442 (2005)

7. $\mathrm{Yu}, \mathrm{SB}, \mathrm{Wu}, \mathrm{HH}, \mathrm{Chen}, \mathrm{JB}$ : Multiple periodic solutions of delayed predator-prey systems with type IV functional responses on time scales. Discrete Dyn. Nat. Soc. 2012, Article ID 271672 (2012)

8. Chen, Y: Multiple periodic solutions of delayed predator-prey systems with type IV functional responses. Nonlinear Anal., Real World Appl. 59, 45-53 (2004)

9. Zhao, K, Ye, Y: Four positive periodic solutions to a periodic Lotka-Volterra predatory-prey system with harvesting terms. Nonlinear Anal., Real World Appl. 11, 2448-2455 (2010)

10. Zhang, ZQ, Tian, TS: Multiple positive periodic solutions for a generalized predator-prey system with exploited terms. Nonlinear Anal., Real World Appl. 9, 26-39 (2007)

11. Li, YK, Zhao, KH, Ye, Y: Multiple positive periodic solutions of $n$ species delay competition systems with harvesting terms. Nonlinear Anal., Real World Appl. 12, 1013-1022 (2011)

12. Gopalsamy, K, Kulenovic, MRS, Ladas, G: Environmental periodicity and time delays in a food-limited population model. J. Math. Anal. Appl. 147, 545-555 (1990)

13. Gourley, SA, Chaplain, MAJ: Travelling fronts in a food-limited population model with time delay. Proc. R. Soc. Edinb. A $132,75-89(2002)$

14. Gourley, SA, So, JW-H: Dynamics of a food-limited population model incorporating non-local delays on a finite domain. J. Math. Biol. 44, 49-78 (2002)

15. Chen, FD, Sun, DX, Shi, JL: Periodicity in a food-limited population model with toxicants and state dependent delays. J. Math. Anal. Appl. 288, 136-146 (2003)

16. Li, YK: Periodic solutions of periodic generalized food limited model. Int. J. Math. Math. Sci. 25, 265-271 (2001)

17. Fang, $\mathrm{H}$ : Multiple positive periodic solutions for a food-limited two-species Gilpin-Ayala competition patch system with periodic harvesting terms. J. Inequal. Appl. 2012, 291 (2012)

18. Gilpin, ME, Ayala, FJ: Global models of growth and competition. Proc. Natl. Acad. Sci. USA 70, 3590-3593 (1973)

19. Lian, B, Hu, S: Asymptotic behaviour of the stochastic Gilpin-Ayala competition models. J. Math. Anal. Appl. 339 419-428 (2008)

20. Lian, B, Hu, S: Stochastic delay Gilpin-Ayala competition models. Stoch. Dyn. 6, 561-576 (2006)

21. He, MX, Li, Z, Chen, FD: Permanence, extinction and global attractivity of the periodic Gilpin-Ayala competition system with impulses. Nonlinear Anal., Real World Appl. 11, 1537-1551 (2010)

22. Zhang, SW, Tan, DJ: The dynamic of two-species impulsive delay Gilpin-Ayala competition system with periodic coefficients. J. Appl. Math. Inform. 29, 1381-1393 (2011)

23. Bohner, M, Peterson, A: Dynamic Equations on Time Scales: An Introduction with Applications. Birkhäuser, Boston (2001)

24. Bohner, M, Peterson, A: Advances in Dynamic Equations on Time Scales. Birkhäuser, Boston (2003)

25. Gaines, RE, Mawhin, JL: Coincidence Degree and Nonlinear Differential Equation. Springer, Berlin (1997)

doi:10.1186/1687-1847-2013-278

Cite this article as: Fang and Wang: Four periodic solutions for a food-limited two-species Gilpin-Ayala type predator-prey system with harvesting terms on time scales. Advances in Difference Equations 2013 2013:278. 